\title{
PERCEPCIÓN DE LOS NIÑOS (AS) DE 7 A 12 AÑOS ACERCA DE LAS DROGAS EN AMÉRICA LATINA ${ }^{1}$
}

Institución: Escuela de Enfermería de Riverao Preto, Universidad de São Paulo

\section{COMO CITAR}

Fernandes, M., Sánchez, O. (2015). Percepción de los niños (as) de 7 a 12 años acerca de las drogas en América Latina. Rev. Enfermería Actual en Costa Rica, 28, 1-12. DOI: http://dx.doi.org/10.15517/revenf.v0i28.17047

\section{RESUMEN}

Introducción. El presente artículo tiene como objetivo analizar la percepción de los niños de 7 a12 años acerca de la temática de las drogas, durante el periodo 1992-2012. uno de los problemas más reiterativos en las poblaciones infantiles es el uso del alcohol, tabaco y demás drogas ilícitas, cuya exposición inicia a edades cada vez más tempranas, motivo por el que es fundamental la participación de equipos de trabajo en conjunto con padres, educadores, psicólogos, psiquiatras, trabajadores sociales, poderes públicos, a lo que debe aunarse una estrategia global de promoción de la salud para enfrentar el fenómeno de las drogas en la niñez.

Métodos. Para desarrollar esta investigación se empleó la revisión bibliográfica integrativa. Para recolectar información se utilizó diversos artículos completos, indexados en bases de datos LILACS y SCIELO, de los que se recuperó 54 artículos, aunque solo diez se relacionan con la temática en estudio.

Resultados. En ninguno de los estudios se estableció diferencia de percepción entre los niños (as) a partir de aspectos como sexo, edad, condiciones económicas, sociales y familiares; no obstante, se relaciona países, sí se detectó diferencias, aunque mínimas, principalmente en lo que respecta al abordaje del tema, el cual se enfoca en el consumo en sí y no en las percepciones que construyen los niños en relación con el consumo

Conclusión. Los niños perciben como inapropiado el consumo de drogas tanto lícitas como ilícitas, puesto que producen múltiples consecuencias que producen alteraciones en la salud y en la apariencia física, así como bajo rendimiento escolar y deterioro en las relaciones familiares y sociales.

Palabras claves: niños, drogas, percepción.

\footnotetext{
${ }^{1}$ Fecha de recepción: 05 de julio del 2014

Fecha de aceptación: 13 de noviembre del 2014

${ }^{2}$ Enfermera. Escola de Enfermagem de Ribeirão Preto da Universidade de São Paulo, Brasil. Correo electrónico: mrcna13@yahoo.com.br.

${ }^{3}$ Enfermera. Escuela de Enfermería de la Universidad de Costa Rica. Costa Rica. Correo electrónico:

OLGA.SANCHEZCHARPENTIER@ucr.ac.cr
} 


\title{
PERCEPTION OF THE CHILDREN FROM 7 TO 12 YEARS ABOUT DRUGS IN LATIN AMERICA ${ }^{1}$
}

Institution: School of Nursing Riverao Preto, University of São Paulo

\author{
Marciana Fernandes Moll ${ }^{2}$ \\ Olga Sánchez Charpentier ${ }^{3}$
}

\section{CITED:}

Fernandes, M., Sánchez, O. (2015). Perception of the children from 7 to 12 years about drugs in Latin America. Rev. Enfermería Actual en Costa Rica, 28, 1-12. DOI: http://dx.doi.org/10.15517/revenf.v0i28.17047

\begin{abstract}
Introduction. This article aims at analyzing the perception of children from 7 to 12 years on the subject of drugs, during the period 1992-2012. one of the most repetitive problems in child populations is the use of alcohol, tobacco and other drugs, whose exhibition starts at increasingly younger ages, reason for which the participation of teams from working together with parents, educators, psychologists, psychiatrists, social workers, public authorities, is essential to a global strategy of health promotion must join to confront the phenomenon of drugs in children.

Methods.To collect information used various full articles indexed in LILACS and SCIELO recovering 54 articles, of which only ten had regarding the issue under study.

Result. In none of the studies was established difference in perception between the children from aspects such as sex, age, family, social and economic conditions; However, relates countries, yes detected differences, although minimal, mainly in regard to the approach to the issue, which focuses on consumption itself and not on perceptions that build children in relation to consumption.

Conclusion. The children perceived as inappropriate drug use both legal and illegal, as they produce multiple consequences, sometimes triggering alterations in health and physical appearance, poor school performance, impaired social and family relations.
\end{abstract}

Key words: children, drugs, perception.

\footnotetext{
${ }^{1}$ Date of receipt:

Date of acceptance: November 13, 2014

${ }^{2}$ Nurse. School of Nursing Ribeirão Preto da Universidade de São Paulo, Brasil. E mail:mrcna13@yahoo.com.br

${ }^{3}$ Nurse. School of Nursing. University of Costa Rica. Costa Rica. E mail: OLGA.SANCHEZCHARPENTIER@ucr.ac.cr
} 


\section{Revista Electrónica Enfermeria Actual de costa Rica}

\section{INTRODUCCIÓN}

Actualmente, en nuestra sociedad, uno de los problemas más reiterativos en las poblaciones infantiles es el uso del alcohol, tabaco y demás drogas ilícitas, cuya exposición inicia a edades cada vez más tempranas, motivo por el que es fundamental la participación de equipos de trabajo en conjunto con padres, educadores, psicólogos, psiquiatras, trabajadores sociales, poderes públicos, a lo que debe aunarse una estrategia global de promoción de la salud para enfrentar el fenómeno de las drogas en la niñez.

Respecto de las drogas lícitas de mayor consumo, el alcohol encabeza la lista (García, Ferriani, 2008), cuyo consumo en la edad adulta si se es hijo de padres alcohólicos: un factor de riesgo más que significativo (Zanoti, 2005). Respecto del panorama mencionado, se considera que el infante tiene ese primer acercamiento con el alcohol, debido a que es una droga lícita, la cual es asequible a través de hábitos culturales desde los que se incita su consumo ya sea por deseos de conocer o por diversión.

Dado que la niñez es un periodo vulnerable de aprendizaje en el que abunda la desinformación en torno a la temáticas sobre drogas, es posible que la ingesta de sustancias lícitas e ilícitas sea prematura, por consiguiente, es necesario establecer medidas preventivas que refuercen las campañas de información de manera que los niños reciban capacitación real basada en investigaciones científicas, para llegar a la adolescencia sin las complicaciones que trae la drogadicción (Rodríguez, 2009).

Entre otras medidas, los programas de prevención del consumo de drogas deben fomentar la autoeficacia, tales como creer en las propias capacidades para organizar y ejecutar las acciones necesarias para manejar situaciones futuras, lo cual favorece el desarrollo de habilidades sociales, la capacidad de enfrentar diferentes situaciones en la vida y la toma de decisiones en los niños, como en el caso del fenómeno de las drogas. Cabe recalcar, que los escolares son el foco de atención de los programas dirigidos a disminuir el consumo de drogas, máxime que lo que sucede en esta etapa de la vida repercute más adelante en las acciones de la adolescencia (Ruíz y Andrade, 2005; Rodríguez, 2009, Melo y Nascimento, 2010).

De igual modo, conviene practicar intervenciones preventivas desde cortas edades, aunque en pocas regiones existen programas suficientemente estructurados que brinden una imagen clara y concluyente sobre las posibilidades de mejora en este campo. Algunas estrategias a futuro son la correcta información y educación para los padres, por medio del desarrollo de medidas educativas, promoción de entornos favorables y reducción de daños, además del desarrollo de estrategias y planes nacionales (Carvajal y De Andrade, 2005). También es imperante crear nuevas políticas públicas con el objetivo de vigilar y exigir el cumplimiento de los derechos de la niñez, adolescencia y juventud en el país, de modo que se opere la toma de decisiones, respecto de mejorar la calidad de vida de este grupo poblacional ( $\underline{\text { Gutiérrez, 2003) }}$.

Otra medida consiste en involucrar a los profesionales de la salud en el desarrollo de todas estas estrategias de prevención, con el objetivo de que impacten en la calidad de vida de los niños (as), sus familias, la escuela y comunidad. Por otra parte, representaría una estrategia para la organización y ejecución de acciones dirigidas a infantes de áreas urbanas y urbano-marginales, sin que haya distinción por el contexto económico en que se desenvuelvan, de manera que decrezca este flagelo social (Gutiérrez, 2003). 


\section{Revista Electrónica Enfermería Actual de costa Rica}

De acuerdo con lo anterior, es posible afirmar que los niños (as) conocen las drogas y están en contacto con estas, por consiguiente, es necesario profundizar en dicha temática y conocer cómo este conocimiento repercute en los ámbitos físico, psíquico y social en sus vidas. A partir de lo mencionado, se plantea como objetivo de este estudio analizar la percepción de los niños y niñas de 7 a 12 años acerca de la temática de las drogas en el periodo 1992-2012.

\section{MATERIALES Y METODOS}

Para desarrollar esta investigación, se eligió la revisión bibliográfica integrativa, fundamentada en los estudios de Ganong (1987), Broome (2000) y Whittemore y Knalf (2005) citado por Melo y otros (2011), la cual se trata de un método de revisión específico que permite incluir diversas líneas de investigación (cuantitativas y cualitativas), que abarca la literatura teórica y empírica, constituida por seis etapas: identificación del problema o cuestionamiento, establecimiento de criterios de inclusión/exclusión de artículos (selección de la muestra), definición de las informaciones que serán extraídas de los artículos seleccionados, análisis de las informaciones, interpretación de los resultados, y presentación de la revisión ( Melo y otros, 2011). De igual modo, incluye el análisis amplio de investigaciones relevantes (Dal Sasso y otros, 2008) que dan soporte a la tomada de decisiones; su finalidad es la búsqueda y síntesis de la percepción de los niños y las niñas en la temática de las drogas.

La pregunta formulada para guiar la revisión integradora fue la siguiente: ¿Cuáles son las evidencias científicas sobre la percepción que tienen los niños (as) acerca de la temática de las drogas, en el periodo 19922012 ?

Para la búsqueda de los artículos científicos, se consultó las bases de datos Literatura Latino-Americana y del Caribe en Ciencias de la Salud (LILACS) y SCIELO, por medio de los descriptores droga, niños, percepción, percepción-niños-drogas, infancia-drogas, efectos físicos, psicológicos y sociales en niños. El levantamiento de las publicaciones en las bases de datos ocurrió en el mes de marzo de 2012.

Los criterios considerados para la inclusión de las publicaciones en el presente estudio, fueron: artículos completos disponibles electrónicamente que abordaran la percepción de los niños de 7 a 12 años acerca de la temática de las drogas sin distinción de nacionalidad; artículos publicados en español, portugués e inglés del periodo 1992-2012.

Fueron recuperados 54 artículos, de los cuales sólo diez tenían relación con los criterios de inclusión anteriormente descritos. La procedencia de los artículos revisados fueron: tres de Brasil; dos de Argentina; dos de Costa Rica; uno de Chile; uno de Ecuador y uno de México.

El análisis y síntesis de los datos se efectuó con una lectura minuciosa, crítica y reflexiva de los artículos, así como de los resultados convergentes o divergentes en relación con el estudio. Los datos extraídos fueron transcritos en un cuadro en el que se agrupó la información de la siguiente manera: la base de datos, título del artículo, año, diseño del estudio, país, población. La exclusión de algunos artículos se debe a que se relacionaban 


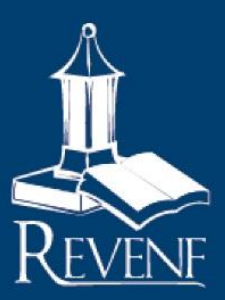

\section{Revista Electrónica Enfermeria Actual de costa Rica}

con el uso y abuso de drogas lícitas e ilícitas en la niñez, así como con el uso de drogas como tratamiento terapéutico o medicamentoso, temáticas que no eran parte de esta revisión bibliográfica.

Los resultados fueron presentados en forma descriptiva, en tres etapas. En la primera se describió los datos de identificación de las publicaciones (autores, año), el país donde se realizó el estudio y el tipo de base de datos donde se encuentra indexado el artículo. En la segunda, se evaluó las características metodológicas de los estudios, clasificados de acuerdo con el diseño del estudio; por último, en la tercera etapa, se describió la percepción que tienen los niños (as) acerca de la temática de las drogas, en el periodo comprendido 1992-2012.

\section{Consideraciones éticas}

En esta investigación se revisó los artículos, se consideró y respetó los derechos de autor, lo cual se evidencia al citar a los autores de cada trabajo en las citas bibliográficas utilizadas como fundamento del artículo.

\section{RESULTADOS}

Se encontró cuatro artículos en la Base de datos LILACS y seis en SCIELO: uno del año 1994; uno del 2003; uno del 2004; tres del 2005; uno del 2008; uno del 2010 y dos del 2011. De los artículos, seis son de tipo cuantitativo con diseños descriptivos, transversales, exploratorios y correlacionales; uno, es cualitativo con diseño etnográfico transversal descriptivo y 1 mixto. Respecto de la población, en nueve de ellos estuvo integrada por escolares de II ciclo de enseñanza primaria y uno con unidades escolares; en cuanto al país de publicación, tres son de Brasil; dos, de Argentina; dos, de Costa Rica; uno, de Chile; uno, de Ecuador y uno, de México. Véase tabla 1. 


\section{Revista Electrónica Enfermería Actual de costa Rica}

Tabla 1

Presentación de artículos en base de datos LILACS Y SCIELO

\begin{tabular}{|c|c|c|c|c|c|}
\hline $\begin{array}{c}\text { Base de } \\
\text { datos }\end{array}$ & Título del artículo & Año & País & Diseño del estudio & Población \\
\hline LILACS & $\begin{array}{l}\text { Opiniones sobre las drogas de los estudiantes de } 6^{\circ} \\
\text { grado de la escuela finca Guararí, Heredia }\end{array}$ & 2011 & $\begin{array}{l}\text { Costa } \\
\text { Rica }\end{array}$ & $\begin{array}{l}\text { Descriptiva- } \\
\text { transversal }\end{array}$ & 114 estudiantes \\
\hline SCIELO & Niños de la calle: Buenos Aires SXXI & 2011 & Argentina & $\begin{array}{l}\text { Etnográfico } \\
\text { transversal } \\
\text { descriptivo }\end{array}$ & $\begin{array}{l}50 \text { niños con edades } \\
\text { de } 8 \text { a } 15 \text { años }\end{array}$ \\
\hline LILACS & $\begin{array}{l}\text { La escuela básica en la prevención del consumo de } \\
\text { alcohol y tabaco: retrato de una realidad }\end{array}$ & 2005 & Ecuador & $\begin{array}{l}\text { Exploratoria } \\
\text { descriptiva }\end{array}$ & $\begin{array}{l}\text { Los alumnos de } \\
5^{\circ}, 6^{\circ} \text { y } 7^{\circ} \text { de } 2 \\
\text { escuelas }\end{array}$ \\
\hline LILACS & $\begin{array}{l}\text { Consumo de drogas lícitas e ilícitas en escolares y } \\
\text { factores de protección y riesgo }\end{array}$ & 2004 & Chile & $\begin{array}{l}\text { Estudio descriptivo, } \\
\text { transversal, } \\
\text { correlacional }\end{array}$ & 301 escolares \\
\hline SCIELO & $\begin{array}{l}\text { Levantamiento del conocimiento y del contacto con } \\
\text { drogas en la comunidad de alumnos de ler, grado de } \\
\text { enseñanza pública y privada en Ribeirão Preto }\end{array}$ & 1994 & Brasil & Empírica & 71 unidades escolares \\
\hline SCIELO & $\begin{array}{l}\text { Uso de drogas en seis a los niños de siete años de edad } \\
\text { de una escuela primaria de Celaya, Guanajuato, México }\end{array}$ & 2008 & México & $\begin{array}{l}\text { Exploratorio } \\
\text { transversal }\end{array}$ & 39 escolares \\
\hline SCIELO & $\begin{array}{l}\text { Evaluando el conocimiento de menores de edad sobre } \\
\text { drogas y alcohol, abuso sexual infantil, violencia } \\
\text { intrafamiliar e infecciones de transmisión sexual: el caso } \\
\text { de la escuela Estados Unidos de América }\end{array}$ & 2010 & $\begin{array}{l}\text { Costa } \\
\text { Rica. }\end{array}$ & Mixto & $\begin{array}{l}180 \text { estudiantes de IV } \\
\text { grado }\end{array}$ \\
\hline LILACS & $\begin{array}{l}\text { O papel das drogas na vida da criança em situação de } \\
\text { rua. }\end{array}$ & 2003 & Brasil & Cualitativa & $\begin{array}{l}\text { Quince niños con } \\
\text { edades entre } 7 \text { y } 12 \\
\text { años }\end{array}$ \\
\hline SCIELO & $\begin{array}{l}\text { La familia y los factores de riesgo relacionados con el } \\
\text { consumo de alcohol y tabaco en los niños y adolescentes } \\
\text { (Guayaquil-Ecuador). }\end{array}$ & 2005 & Ecuador & $\begin{array}{l}\text { Descriptivo- } \\
\text { exploratorio }\end{array}$ & $\begin{array}{l}139 \text { familias de niños } \\
\text { con edades entre } 8 \text { y } \\
12 \text { años }\end{array}$ \\
\hline SCIELO & $\begin{array}{l}\text { Consumo de sustancias psicoactivas en niños } \\
\text { escolarizados del conurbano Bonaerense durante el año } \\
2004\end{array}$ & 2005 & Argentina & Exploratorio & $\begin{array}{l}\text { Niños y niñas entre } \\
10 \text { y } 12 \text { años, que } \\
\text { concurren a } 5^{\circ}, 6^{\circ} \text { y } \\
7^{\circ} \text { año }\end{array}$ \\
\hline
\end{tabular}

En lo que respecta a la percepción de los niños (as) acerca de la temática de las drogas, afirman que la droga es dañina para la salud y las personas, así como que son sustancias malas que matan, que perjudican la salud de quien la usa y que se debe eliminarlas (Ferriani y otros, 1994; Araya, Arce, Arroyo, Cabezas, Curcó y González,2011; García y Ferriani 2008; Ruíz y Andrade, 2005).

Para los niños fumar, andar con malas compañías, oler cola, aplicarse inyección, es sinónimo de "estar loco"; además, consideran que el hachís es la droga más peligrosa y que la cocaína y el polvo blanco son una droga que mata las personas y las enloquece hasta el punto de que pueden atacar a los padres. Los consideran que la única sustancia aceptable es el tabaco envuelto en papel de cigarrillo (Ferriani, Cano, Spagnol y Silva, 1994). 


\section{Revista Electrónica Enfermeria Actual de costa Rica}

Según dicho autor, los niños desaprueban el consumo de alcohol, opinión que aumenta si se trata de sustancias ilegales, puesto que dañan la salud (Slapak y Grigoravicius, 2006). De acuerdo con el estudio, cuando a los niños (as) participantes se les ofreció probar las sustancias, respondieron con desagrado, y afirmaron no querer repetir esa "desagradable experiencia"; incluso, hubo niños (as) que definitivamente rechazaron "probar" el cigarro y las bebidas alcohólicas. (García y Ferriani, 2008).

A pesar de lo anterior, en otro estudio se detecta una tolerancia social respecto del consumo de sustancias en gran parte de las familias de origen de los niños, quienes comentan que sus padres y hermanos ingieren drogas como la cerveza, pastillas, proxi, entre otras (Brasesco, 2011).

En relación con las percepciones que tienen acerca del perjuicio de las drogas, expresan que el tabaco es algo malo, que perjudica el embarazo, que si se está al lado de alguien que fuma es como si uno fumara, aparte de que es malo para el medioambiente y para los niños (Ferriani y otros, 1994).

El conocimiento que tienen los niños acerca de las drogas denota cuán expuestos están a este tema; por ejemplo, saben respecto la marihuana, cocaína, polvo de cocaína, cola, hachís, cigarrillo, lápiz tóxico (Ferriani y otros, 1994; Josephy y otros, 2010). En relación con lo anterior, se puede afirmar que los niños consideran que la droga daña la salud, desestructura la familia, provoca muerte, así como muchos perjuicios para la persona, la familia y la sociedad. Autores como Carvajal y Andrade (2005), y Araya y otros (2011), aclaran que algunos niños consumen drogas por curiosidad, por probar, buscar placer, por presión de los amigos, por buscar nuevas experiencias o simplemente porque todos lo hacen.

Respecto de las consecuencias que origina el consumo de drogas en la salud, se menciona la pérdida de peso, la coloración de los dientes amarillos, quedarse sin sangre, pérdida de la voz y el no poder correr como antes. Otros mencionan que se atenúa el frío , no se siente hambre, se produce dolor de cabeza, palpitaciones, visión lenta, así como que disminuye el dolor (Araya y otros, 2011; Brasesco, 2011).

En cuanto a los efectos psicológicos de las drogas en los niños, se incluye el sentir un falso bienestar temporal, razón por la que siguen consumiendo, aunque luego vuelven a su rutina y sienten tristeza y ganas de llorar (Duvicq, Pereira y Carvalho, 2004); por otra parte, aun cuando consideran el tabaco como un relajantetranquilizador, pueden llegar a oír voces, presentar sentimientos de omnipotencia y excitación, de ahogo, de que enloquecen, de que todo lo que sucede es gracioso, razón por la pierden el miedo y sufren accidentes. Además de tener sensaciones de analgesia y anestesia, un niño (a) refirió que se fracturó y no sintió nada (Brasesco, 2011), mientras que otros sienten "bronca" o "vergüenza" por el estado en que se encuentran (Slapak y Grigoravicius, $\underline{2005})$.

En relación con los efectos sociales de las drogas sobre los niños (as), ellos perciben que perjudica no solo la salud sino a la comunidad donde viven o donde se drogan (Ferriani y otros, 1994), parte de que el adicto se convierte en una amenaza para otros, debido a que dañan objetos ajenos, roban, discuten con la familia, se ausentan de la escuela y son irresponsables en sus deberes escolares (Duvicq, Pereira y Carvalho, 2004). 


\section{Revista Electrónica Enfermeria Actual de costa Rica}

De igual modo, los niños afirman que quienes consumen drogas son maltratados en muchas ocasiones por la familia, compañeros, personas de la calle y por la policía, máxime si producen problemas (Ribeiro, Aparecida, Silva y Barros, 2003; Slapak y Grigoravicius, 2006).

\section{DISCUSIÓN}

Después de la revisión de los artículos se encontró que existe mucha literatura relacionada a los niños (as) que consumen drogas, no así de la percepción que tienen sobre el consumo y sus efectos sobre la salud.

Algunas causas que aumentan el riesgo de que en la niñez se consuma drogas a temprana edad (García, Ferriani, 2008) es el ausentismo escolar, el desviarse a comprar tabaco, que algún familiar consuma drogas y, peor aún, si lo hace enfrente de los infantes y les permiten probarlas (Luque, Mesas, Alberro, Palma, Ortíz, Cáliz y Gálvez, 2002), también la falta de cariño y atención puede desembocar en consumo de drogas (Ribeiro y otros 2003); por consiguiente, en este contexto es esencial que los niños construyan un concepto de sí mismos y disminuyan el ausentismo escolar (Carvajal y Andrade, 2005),

En cuanto a las estadísticas, Grant, citado por Zanoti y Carvalho (2005), confirmaron que, en niños y adolescentes menores de dieciocho años, uno de cada cuatro está expuesto a consumir alcohol en el ambiente familiar, información que se investigó considerando variables como la autoestima, la edad, el género y el uso de alcohol de donde se extrajo que un 52\% ya abusaba del alcohol antes de los 18 años. Acerca del tema, los autores convergen en que es imprescindible investigar más sobre esta influencia y las repercusiones del alcoholismo familiar en los niños (as) y adolescentes, debido a que no hay muchos estudios en el país y, menos aún, en el mundo.

Otros aspectos a tomar en cuenta son los riesgos a los que pueden estar expuestos los niños y niñas cuando tiene el acceso a las drogas tanto lícitas como ilícitas, sea por el consumo de sus padres, madres y/o familiares, que los predispone a consumir y vender tales sustancias (Luque y otros, 2002; García y Ferriani 2008), con el fin de subsistir y entrar en pandillas (Carvajal y Andrade, 2005), lo cual aumenta su vulnerabilidad (Ribeiro y otros, $\underline{2003}$.

En otro trabajo, Ruiz y Andrade (2005) detectaron que en un 52\% del total de las familias encuestadas se acepta el consumo de alcohol y tabaco, lo cual agrava la situación, debido a que el niño aprende por lo que ve y asimila, de modo que cuando crezca es posible que haga lo mismo, razón por la que el comportamiento de los padres representa un modelo para sus hijos. Por otra parte concluyen que la familia, escuela y medio social son tres elementos del sistema social que están en constante comunicación, por ello cualquier situación repercutirá en el conjunto del sistema en el que la familia es el mejor agente de socialización, dado que en esta se desarrolla normas de conducta, costumbres, valores dominantes de la sociedad, modelos en general y la interpretación de los modelos en función a la clase social, cultura y subcultura a los que pertenece, lo cual influye en el aprendizaje de los niños, quienes asumen roles, hábitos, normas, costumbres, actitudes en general y tradiciones de la sociedad.

Al mismo tiempo, el consumo de drogas en niños (as) se relaciona con encontrarse solos en la mayoría de las actividades, lo cual se constituye en un riesgo de experimentar con alcohol y otras drogas (Rodríguez, Hernández, Fernández, 2006), máxime que para algunos niños iniciarse en el consumo de drogas puede originarse 


\section{Revista Electrónica Enfermería Actual de costa Rica}

por diversión (Araya y otros, 2011); según Ballester y Gil (2002) el 16\% de niños, entre 9 y 14 años, consumen alcohol los fines de semana junto con sus amigos y entre un 11-13\% se ha embriagado entre alguna vez y muchas veces, además que se detecta algunas actitudes excesivamente optimistas acerca de los efectos del alcohol, mas no se considera cuán vulnerable es esta población. Otro aspecto importante es la asociación entre consumo de alcohol y diversión o relaciones sociales que ya se da en estas edades tempranas. A pesar de lo anterior, según algunas investigaciones, las campañas preventivas han contribuido a que muchos niños se nieguen a consumir drogas (Luque y otros, 2002).

En ninguno de los estudios se estableció diferencia de percepción entre los niños (as) a partir de aspectos como sexo, edad, condiciones económicas, sociales y familiares; no obstante, se relaciona países, sí se detectó diferencias, aunque mínimas, principalmente en lo que respecta al abordaje del tema, el cual se enfoca en el consumo en sí y no en las percepciones que construyen los niños en relación con el consumo.

Frente a esa realidad, es imprescindible fomentar la preparación y conocimiento de los niños acerca de temas relacionados con el fenómeno de las drogas, sus implicaciones físicas, mentales y sociales (Josephy y otros, $\underline{2010})$.

\section{CONCLUSION}

Los niños (as) perciben el consumo de drogas tanto lícitas como ilícitas como inapropiado, debido a que producen múltiples consecuencias que alteran la salud y apariencia física y provocan bajo rendimiento escolar, deterioro en las relaciones familiares y sociales.

La exposición a las diferentes drogas (principalmente las lícitas) y el inicio del consumo a edades muy tempranas para la población infantil empieza en el hogar, donde hay familiares que las ingieren (padres, madres, tíos, hermanos, etc.), y que muestran cierta "tolerancia social" acerca del tema, lo cual genera que muchos niños consideren el consumo como un acto natural en su hogar y contexto social. Sin embargo, según las investigaciones, un factor positivo es que la población infantil rechaza las drogas ilícitas en la mayoría de las ocasiones, mas no sucede lo mismo con las lícitas, las cuales son más accesibles, de uso más frecuente y "aceptable socialmente" dentro de los grupos familiares.

La niñez es una etapa en la que la curiosidad y el descubrir nuevas experiencias unido a la presión de los pares y la cotidianidad en la exposición o acceso a las drogas, puede incitar un inicio temprano del uso de las drogas lícitas e ilícitas, vivencia que afecta a la persona involucrada y a la comunidad donde vive y se desarrolla.

Del abordaje de la temática del fenómeno de las drogas en los diferentes espacios donde se desenvuelven los niños (as) (escuelas, centros recreativos, actividades comunitarias, actividades extracurriculares, lugares de cuido, entre otros) se obtiene información veraz y relevante del contexto familiar y social de los infantes, a partir de lo que ellos perciben los diferentes riesgos del uso y abuso de las drogas, aunque la exposición sea inherente a su cotidianidad. 


\section{Revista Electrónica Enfermeria Actual de Costa Rica}

\section{REFERENCIAS BIBLIOGRÁFICAS}

Añaños, F , Añaños, K, Añaños, C, Arroyo, S. (2010). Realidades y representaciones sociales de las niñas y los niños de la calle en Perú. Revista española de drogodependencias 2, 182-194. Recuperado de:

http://dialnet.unirioja.es/servlet/articulo?codigo $=3281061$

Araya, G., Arce, G., Arroyo, S., Cabezas, C., Curcó, R., González, E. (2011). Opiniones sobre las drogas de los estudiantes de $6^{\circ}$ grado de la escuela Finca Guararí, Heredia. Med. leg. Costa Rica 28 (1): 45-54.

Recuperado de http://www.scielo.sa.cr/scielo.php?script=sci_arttext\&pid=S1409-00152011000100006\&lng=es.

Ballester, R., Gil, M. (2002). Inicio temprano de consumo de alcohol entre niños de 9 a 14 años). Análisis y Modificación de Conducta 28 (118)165-212. Recuperado de: https://www.google.com/\#hl=es\&output=search\&sclient=psy-

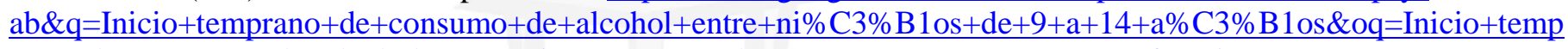
rano+de+consumo+de+alcohol+entre+ni\% $3 \% \mathrm{~B} 10 \mathrm{~s}+\mathrm{de}+9+\mathrm{a}+14+\mathrm{a} \% \mathrm{C} 3 \% \mathrm{~B} 1$ os $\& a q=f \& a q i=\mathrm{g}-$

v1\&aql=\&gs_l=hp.12..0i15.184951184951012259611111010101011511115111811110.frgbld.\&pbx=1\&bav=on.2,or.r_gc.r_pw.r_qf.,cf.osb\&fp=e5f67c6812b76d1e\&biw=1366\&bih=667

Brasesco M. (2011). Infancia marginal en las grandes ciudades: Los niños y niñas de la calle . Actas de Jornadas de Investigación. 1. Recuperado de: https://www.google.com/\#hl=es\&sclient=psyab\&q=INFANCIA+MARGINAL+EN+LAS+GRANDES+CIUDADES\%3A+LOS+NINOS+Y+NINAS+DE+LA+C ALLE.\&oq=INFANCIA+MARGINAL+EN+LAS+GRANDES+CIUDADES:+LOS+NINOS+Y+NINAS+DE+LA+ CALLE.\&aq $=\mathrm{f} \& \mathrm{aqi}=\& \mathrm{aql}=\& \mathrm{gs} \quad \mathrm{l}=\mathrm{hp} .12 \ldots 8405101840510111841878111110101010101011010$. frgbld $. \& \mathrm{pbx}=1 \& \mathrm{bav}=\mathrm{on} .2, \mathrm{o}$ r.r_gc.r_pw.r_qf.,cf.osb\&fp=e5f67c6812b76d1e\&biw=1366\&bih=667

Brasesco, M. (2011). Niños de la calle: Buenos Aires SXXI. Cienc. Psicol. 5(1) 7-18. Recuperado de: http://www.scielo.edu.uy/scielo.php?script=sci arttext\&pid=S1688-40942011000100002\&lng=es.

Carvajal, L., Andrade, D. (2005). La escuela básica en la prevención del consumo de alcohol y tabaco: retrato de una realidad. Revista Latino-Americana de Enfermagem, 13(spe), 784-789. Recuperado de:

http://www.scielo.br/scielo.php?script=sci_arttext\&pid=S0104-11692005000700004\&lng=es\&tlng=es. http://dx.doi.org/10.1590/S0104-11692005000700004.

Dal Sasso, K., Campos, Renata., Galvao, C. (2008). Revisão integrativa: método de pesquisa para a incorporação de evidências na saúde e na enfermagem. Red de Revistas Científicas de América Latina, el Caribe, España y Portugal. 17 (4) 758-764. Recuperado de: http://redalyc.uaemex.mx/redalyc/pdf/714/71411240017.pdf

Duvicq, G., Pereira, N., Carvalho, A. (2004). Consumo de drogas lícitas e ilícitas en escolares y factores de protección y riesgo. Rev. Latino-am Enfermagem 12(spe)345-51. Recuperado de: http://www.scielo.br/scielo.php?pid=S0104$\underline{11692004000700008 \& \text { script }=\text { sci abstract\&tlng=es }}$

Ferriani, M., Cano, M., Spagnol, C., Silva, S. (1994). Levantamiento del conocimiento y del contacto con drogas en la comunidad de alumnos de 1er grado de enseñanza pública y privada en Ribeirão Preto. Rev. Latino-Am. Enfermagem 2(2) 55-72. Recuperado de: http://www.scielo.br/scielo.php?script=sci arttext\&pid=S0104$11691994000200005 \& \operatorname{lng}=$ es. http://dx.doi.org/10.1590/S0104-11691994000200005. 


\section{Revista Electrónica Enfermeria Actual de Costa Rica}

García, M., Ferriani, M. (2008). Uso de drogas en seis niños a los siete años de edad de una escuela primaria de Celaya, Guanajuato, México. Revista Latino-Americana de Enfermería, 16 (spe) 523-528. Recuperado de:

http://www.scielo.br/scielo.php?script=sci_arttext\&pid=S0104$11692008000700004 \& \operatorname{lng}=$ en\&tlng=en.10.1590/S0104-11692008000700004.

Gutiérrez, C. (2003). Adicción a drogas: consumo y consecuencias en continuo cambio. An. Med. Interna (Madrid); 20(10): 5-7. Recuperado de: http://scielo.isciii.es/scielo.php?script=sci_arttext\&pid=S0212-

71992003001000001\&lng=es.

http://dx.doi.org/10.4321/S0212-71992003001000001.

http://www.revistasusp.sibi.usp.br/scielo.php?script=sci arttext\&pid=S141591042003000200005\&lng=pt\&nrm=iso\&tlng=pt

Josephy, S., Mora, R., Ramos, R., Sequeira, A., Vázquez, M., Zumbado D. ( 2010). Evaluando el conocimiento de menores de edad sobre drogas y alcohol, abuso sexual infantil, violencia intrafamiliar e infecciones de transmisión sexual: el caso de la Escuela Estados Unidos de América; Latino-am Enfermagem 27(2): 27-33.

Recuperado de: http://www.scielo.sa.cr/scielo.php?script=sci_arttext\&pid=S1409-00152010000200003\&lng=es.

Luque M., Mesas, A., Alberro, J., Palma, S., Ortiz, F., Cáliz, A., Gálvez, L. (2002). ¿Qué piensan los niños sobre el tabaco? Medicina de Familia (And) 3 (3). Recuperado de: http://samfyc.es/Revista/PDF/v3n3/06.pdf

Medina, M., Cravioto, M., VIillatoro, J., Fleiz, C., Galván, F., Tapia, R. (2003). Consumo de drogas entre adolescentes: resultados de la Encuesta Nacional de Adicciones, 1998. Salud Pública De México 45 (1).

Recuperado de: http://www.scielo.org.mx/pdf/spm/v45s1/15442.pdf

Melo, D., Nascimento, L. (2010). Hacia autoeficacia y actitud del consumo de drogas en la infancia: explorando los conceptos. Revista Latino-Americana de Enfermería , 18 (SPE), 655-662. Recuperado de:

http://www.scielo.br/scielo.php?script=sci_arttext\&pid=S0104$\underline{11692010000700024 \& \operatorname{lng}=e n \& \operatorname{lng}=\text { es. } 10.1590 / \text { S0104-11692010000700024. }}$

Melo, M.,, Barbosa, M., Souza, P. (2011). Satisfacción en el trabajo del equipo de enfermería: revisión integradora. Revista Latino-Americana de Enfermagem, 19(4), 1047-1055. Recuperado de: http://www.scielo.br/scielo.php?script=sci_arttext\&pid=S0104-11692011000400026\&lng=en\&tlng=es. 10.1590/S0104-11692011000400026.

Ribeiro, M., Aparecida, M., Silva, A., Barros, S. (2003). O papel das drogas na vida da criança em situação de rua. Rev. Ter. Ocup. Univ. São Paulo 14 (2) 79-84. Recuperado de: http://www.revistasusp.sibi.usp.br/scielo.php?script=sci arttext\&pid=S141591042003000200005\&lng=pt\&nrm=iso\&tlng=pt

Rodríguez, J., Fernández A., Hernández, E., Valdés, M., Villalón, M., Ramírez, S. (2009). Estudio de prevalencia del consumo de drogas en escolares de Enseñanza Básica, a través de la metodología de pares. Rev. Méd 137(6) 774-784. Recuperado de http://www.scielo.cl/scielo.php?script=sci_arttext\&pid=S0034-98872009000600007\&lng=es doi: 10.4067/S0034-98872009000600007. 


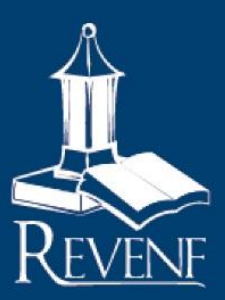

\section{Revista Electrónica Enfermeria Actual de costa Rica}

Rodríguez, J., Hernández, E., Fernández, A. (2007). Descripción del consumo de drogas lícitas e ilícitas por género a través de la metodología de pares. Rev. Méd 135(4) 449-456. Recuperado de: http://www.scielo.cl/scielo.php?script=sci_arttext\&pid=S0034-98872007000400006\&lng=es. doi: 10.4067/S0034-98872007000400006.

Ruiz, M. y Andrade, D. (2005). La familia y los factores de riesgo relacionados con el consumo de alcohol y tabaco en los niños y adolescentes (Guayaquil-Ecuador). Rev. Latino-Am. 813-818.

Recuperado de: http://www.scielo.br/scielo.php?script=sci_arttext\&pid=S0104-11692005000700008\&lng=es. http://dx.doi.org/10.1590/S0104-11692005000700008.

Slapak, S., Grigoravicius, M. (2006). Consumo de sustancias psicoactivas en niños escolarizados del conurbano bonaerense durante el año 2004. Anu. investig.13. Recuperado de:

http://www.scielo.org.ar/scielo.php?script=sci arttext\&pid=S1851-16862006000100023\&lng=es.

Slapak, S., GrigoraviIcius, M. (2005). Estudio exploratorio sobre la presencia del consumo de sustancias psicoactivas en niños entre 10 y 12 años y en sus padres o adultos responsables. Anu.

investig.12

Recuperado de:

http://www.scielo.org.ar/scielo.php?script=sci_arttext\&pid=S1851-16862005000100015\&lng=es.

Zanoti, D. y Carvalho, A. (2005). Alcoolismo parental e suas repercussões sobre crianças e adolescentes: uma revisão bibliográfica. SMAD, Rev. Eletrônica Saúde Mental Álcool Drog. 1(2).

Recuperado de: http://www.revistasusp.sibi.usp.br/scielo.php?script=sci arttext\&pid=S1806-

$\underline{69762005000200007 \& \operatorname{lng}=\text { pt. }}$ 